\title{
Astérisque
}

\section{ALAN DANKNER}

\section{On smale's axiom a dynamical systems}

\author{
Astérisque, tome 49 (1977), p. 19-22 \\ <http://www.numdam.org/item?id=AST_1977_49_19_0>
}

C Société mathématique de France, 1977, tous droits réservés.

L'accès aux archives de la collection « Astérisque » (http://smf4.emath.fr/ Publications/Asterisque/) implique l'accord avec les conditions générales d'utilisation (http://www.numdam.org/conditions). Toute utilisation commerciale ou impression systématique est constitutive d'une infraction pénale. Toute copie ou impression de ce fichier doit contenir la présente mention de copyright.

\section{NumDam}

Article numérisé dans le cadre du programme Numérisation de documents anciens mathématiques http://www.numdam.org/ 


\section{ON SMALE'S AXIOM A DYNAMICAL SYSTEMS*}

by Alan Dankner

Introduction. Let $f$ be a diffeomorphism of a compact manifold $M$ and $\Omega(f)$ be the nonwandering set of $f$. If $\Omega(f)$ is hyperbolic, are the periodic points of $f$ dense in $\Omega(f)$ ? That is, does Axiom $A(a)$, hyperbolicity of $\Omega(f)$, Imply Axiom $A(b)$, denseness of the periodic points in $\Omega(f)$ ? The question was posed by Smale in [3] and answered affirmatively, if $M$ is 2-dimensional, by Newhouse and Palis in [2]. Beyond dimension two, the answer is no.

Theorem. There is a diffeomorphism of $\mathrm{s}^{3}$, the 3-sphere, with an infinite hyperbolic nonwandering set, but a finite set of periodic points.

I would like to thank M. Hirsch and J. Guckenhelmer for useful conversations while preparing this paper. Special thanks are due J. Palis, who introduced the problem to me, and C. Pugh, my thesis advisor, for his encouragement and help.

Definitions. Given $\mathrm{f} \in \operatorname{Diff}(\mathrm{M}), \Omega=\Omega(\mathrm{f})$, the nonwandering set of $f$, consists of all $x \in M$ such that given $U$, a neighborhood of $x$, there is an $n>0$ such that $f^{n}(U) \cap U \neq \emptyset$.

An f-invariant set $\Lambda$ has a hyperbolic stmucture if there is a continuous, Tf-invariant splitting of the tangent bundle restricted to $\Lambda, T M=E^{S} \oplus E^{u}$, such that $T f$ expands vectors in $E^{\mu}$ and contracts vectors in $E^{S}$. That is, there are $c>0,0<\lambda<1$, and a Riemannian metric $\|\cdot\|$, such that for $n \geq 0$, $\left\|\mathrm{Tf}^{\mathrm{n}} \mathrm{v}\right\| \leq c \lambda^{\mathrm{n}}\|v\|$ if $v \in E^{s}$ and $\left\|\mathrm{Tf}^{-\mathrm{n}} \mathrm{v}\right\| \leq \mathrm{c} \lambda^{\mathrm{n}}\|\mathrm{v}\|$ if $\mathrm{v} \in \mathrm{E}^{\mathrm{u}}$. ${ }^{\star}$ AMS (MOS) Subject Classifications (1970). Primary 58F15 


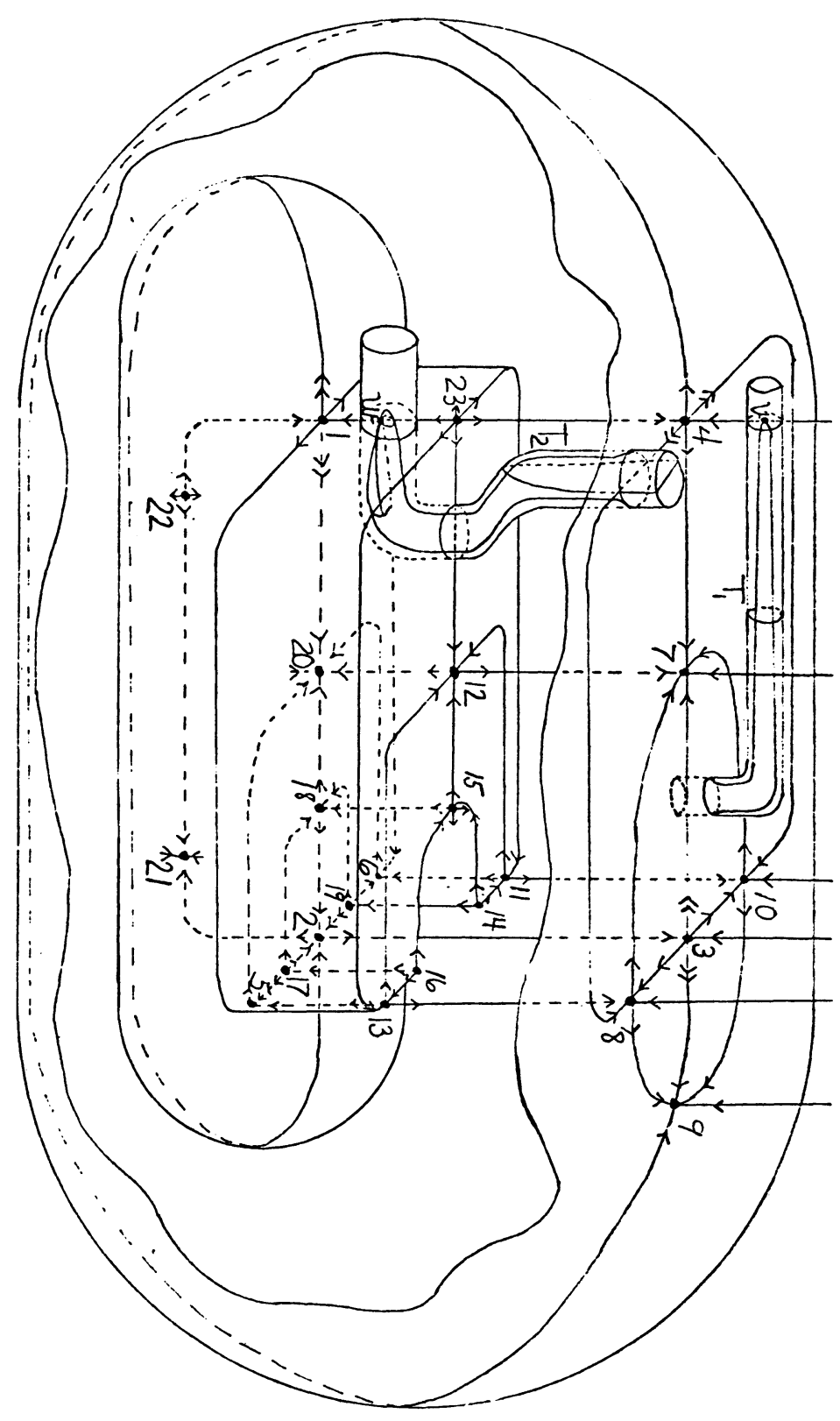


f satisfies Axiom A if

(a) $\Omega(f)$ has a hyperbolic structure, and

(b) the periodic points are dense in $\Omega(f)$.

Additional information may be found in [3].

Sketch of the Proof. The example is constructed first in $\mathbb{R}^{3}$, then extended to $s^{3}$ by adding a point source at infinity. The figure shows the 23 hyperbolic fixed points of the diffeomorphism and many of their stable and unstable manifolds. These invariant manifolds form two concentric 2-spheres. (Part of the outer one is cut away.) The "finger" in $T_{1}$ is formed by pushing $\mathrm{W}^{\mathrm{u}}\left(\mathrm{p}_{3}\right)$ to meet $\mathrm{W}^{\mathrm{S}}\left(\mathrm{P}_{4}\right)$ non-transversely at $\mathrm{v}$. The "finger" in $\mathrm{T}_{2}$ is formed by pushing $\mathrm{W}^{\mathrm{u}}\left(\mathrm{p}_{4}\right)$ to meet $\mathrm{W}^{\mathrm{s}}\left(\mathrm{p}_{1}\right)$ transversely at two points; one of them is labeled w. It can be verified that there is an $\mathrm{f} \in \operatorname{Diff}\left(\mathbb{R}^{3}\right)$ with $\Omega(f)=\bigcup_{i=1}^{23} \mathrm{p}_{1}$.

The goal is to modify $f$ to a new diffeomorphism $\tilde{f}$ such that $\Omega(\tilde{f})=\bigcup_{1=1}^{23} p_{1} \cup \underset{n \in \mathbb{Z}}{\cup} \tilde{f}^{n}$. If such an $\tilde{\mathrm{f}}$ exists, the theorem is proved because the transversality at $w, W^{u}\left(p_{4}\right) \hbar_{w} W^{s}\left(p_{1}\right)$, allows us to define a hyperbolic structure on $\Omega(\tilde{f})$.

$w$ is made nonwandering by adding to $f$ a rotation whose support is contained in a thin, short cylinder about its axis of rotation, $\mathrm{w}^{\mathrm{u}}\left(\mathrm{p}_{2}\right) \cap \mathrm{w}^{\mathrm{s}}\left(\mathrm{p}_{3}\right)$. Using Cloud Lemma arguments, one can show there are points arbitrarily near $w$ that travel around the inner sphere, are rotated part way around $\mathrm{w}^{\mathrm{u}}\left(\mathrm{p}_{2}\right) \cap \mathrm{w}^{\mathrm{s}}\left(\mathrm{p}_{3}\right)$, pass through the "finger" in $\mathrm{T}_{1}$, reach the "finger" in $\mathrm{T}_{2}$, and return near w. The difficult part of the argument is to show $\Omega(\tilde{\mathrm{f}}) \subset \bigcup_{i=1}^{23} \mathrm{p}_{1} \cup \underset{\mathrm{n} \in \mathbb{Z}}{\cup} \tilde{\mathrm{f}}^{\mathrm{n}} \mathrm{w}$ : for example, to show $\mathrm{v} \notin \Omega(\tilde{\mathrm{f}})$. (If $v \in \Omega(\tilde{f})$, then hyperbolicity demands that $W^{u}\left(p_{3}\right) \hbar_{v} W^{s}\left(p_{4}\right)$. 
This eventually leads to a transverse homoclinic point and Infinitely many periodic points, contrary to the goal of the theorem.) The rotation must be constructed very carefully and "adjusted" by a further modification of $f$ in order to avoid extra $\Omega$. This requires delicate, non-generic analysis which will appear elsewhere along with a more detailed exposition of the entire construction of $\tilde{\mathrm{f}}$.

Remarks. Diffeomorphisms with the properties of the example can be found in every isotopy class of Diff(M) for any compact manifold $M$ of dimension greater than two. Suspension yields flows with the corresponding properties on all compact manifolds of dimension greater than three, but whether Axiom A(a) implies Axiom $A(b)$ remains an open question for flows on 3-manifolds.

In [1] it is shown that hyperbolicity of the chain recurrent set of a flow implies the flow satisfies Axiom $A$ and the no cycle property. This contrasts sharply with our results.

\section{References}

[1] R. Churchill, J. Franke, and J. Selgrade, "A geometric criterion for hyperbollcity of flows," Proc. Amer. Math. Soc. 62 (1977) 137-143.

[2] S. Newhouse and J. Palis, "Hyperbolic nonwandering sets on two-dimensional manifolds," in Proc. Salvador Symp. on Dynamical Systems 1971, Academic Press, New York (1973), 203-301.

[3] S. Smale, "Differentiable dynamical systems," Bulz. Amer. Math. Soc. 73 (1967) 757-817. 